RESEARCH ARTICLE

\title{
Adaptive tower damping control for offshore wind turbines
}

\author{
Valentin Pascu ${ }^{1,2}$, Stoyan Kanev ${ }^{1}$ and Jan-Willem van Wingerden ${ }^{2}$ \\ 1 Wind Energy Unit, Energy Research Centre of the Netherlands, 1755 LE Petten, The Netherlands \\ 2 Delft Center for Systems and Control, Technische Universiteit Delft, 2628 CD Delft, The Netherlands
}

\begin{abstract}
This paper deals with the problem of wind turbine tower damping control design and implementation in situations where the support structure parameters vary from their nominal design values. Such situations can, in practice, occur for onshore and especially offshore wind turbines and are attributed to aging, turbine installation, scour or marine sand dunes phenomena and biofouling. Practical experience of wind turbine manufacturing industry has shown that such effects are most easily quantified in terms of the first natural frequency of the turbine support structure. The paper brings forward a study regarding the amount to which nominal tower damping controller performance is affected by changes in the turbine natural frequency. Subsequently, an adaptive tower damping control loop is designed using linear parameter-varying control synthesis; the proposed tower damping controller depends on this varying parameter which is assumed throughout the study to be readily available. An investigation of the fatigue load reduction performance in comparison with the original tower damping control approach is given for a generic three-bladed horizontal-axis wind turbine. Copyright @ 2016 John Wiley \& Sons, Ltd.
\end{abstract}

\section{KEYWORDS}

wind turbine control; tower damping control; linear parameter-varying control; adaptive control; load mitigation

\section{Correspondence}

Valentin Pascu, Wind Energy Unit, Energy Research Centre of the Netherlands,1755 LE Petten, The Netherlands.

E-mail: valentin.c.pascu@gmail.com

Received 9 December 2015; Revised 4 April 2016; Accepted 30 August 2016

\section{INTRODUCTION}

Wind power has proven to be one of the most versatile forms of renewable energy. ${ }^{1}$ Given the massive potential at sea, offshore wind energy is currently emerging as the most promising method of achieving the global targets for wind energy production. $^{2}$ The cost of offshore energy is, however, still too high and strongly relies on governmental subsidies. In order to make it a competitive source of energy and speed up the envisioned large scale implementation, lots of research aiming at lowering the cost of offshore wind energy is being performed. Among the many other technological advances being made, wind turbine control plays an important role to lowering the cost of offshore energy by reducing the turbine loads and increasing the reliability of wind turbines.

Offshore wind turbines operate in harsh, time-varying environmental conditions resulting in significant uncertainties during the design. More specifically, wind turbine manufacturers face the problem that the support structure modal parameters (such as frequency and damping) deviate significantly from their design values. ${ }^{3,4}$ Even more, these parameters also vary with time because of e.g., scour, formation of marine sand dunes and biofouling. ${ }^{1,5-8}$ As the true support structure frequency is not exactly known in advance, is time-varying and deviates from one turbine in the farm to another, a robust design of the support structure is required to ensure it can withstand the worst-case loading that may occur during its lifetime. Even more, the wind turbine controller, tuned for a wrong support structure frequency, is not only expected to perform sub-optimally but might even increase the loads on the support structure as shown later on in the paper. This leads altogether to conservative designs of the support structure with increased material costs and questionable reliability.

In an attempt to reduce the costs because of this design and operational uncertainty, in this paper an adaptive control algorithm is developed that reduces the loads on the support structure in a worst-case sense in the presence of uncertainty in the support structure frequency. This parameter is assumed to be unknown during the design apart from a realistic possible range of variation, very slowly-varying and online-available (i.e., identifiable using measured data, for one possible 
approach see e.g., Balaguer et al. ${ }^{9}$ ). Given this setting, the objective of this paper is to develop a methodology for the design of a control algorithm that adapts its parameters based on the tower frequency, estimated by some means. The purpose is to achieve improved performance in terms of reduction of fatigue loads on the support structure when compared with conventional control where the controller is tuned for the nominal (design) value of the tower frequency. Based on a detailed analysis, the focus of this work is to put on the active fore-aft tower damping control loop as this loop suffers most by the considered changes in the tower dynamics. Damping of the fore-aft vibrations of the tower is achieved, as usual, by controlling the thrust force on the rotor by means of collectively pitching the blades based on the measured tower top acceleration. ${ }^{10}$

For addressing the aforementioned parameter-dependent control design problem, the well-established framework for the design and analysis of linear parameter-varying (LPV) controllers (see e.g., other studies ${ }^{11-13}$ ) is very suitable. Generally speaking, an LPV system is a state-space model that depends on either its state vector (after the linearization procedure of a non-linear system) or on some external parameter that defines its operating condition (e.g., the wind input in a wind turbine model or the support structure frequency). Either way, when this parameter defining the system dynamics is known, an LPV controller that depends on the same parameter (called scheduling parameter) can be designed and is less conservative (i.e., achieves better control performance) than a linear time-invariant controller (be it a nominal controller designed for the nominal value of the scheduling parameter or a robust controller designed to optimize the performance for the worst-case value of the scheduling parameter). The performance of LPV controllers is typically selected as some system norm (usually either the $\mathcal{H}_{2}$ or the $\mathcal{H}_{\infty}$ norm, see e.g., Skogestad and Postlethwaite ${ }^{14}$ ). When it comes to load reduction controls for wind turbines, the performance is typically evaluated in terms of fatigue equivalent loads; however, fatigue loads evaluation involves time-domain simulations and cannot be explicitly incorporated into a performance criterion that can be handled directly by modern control theory. For that reason, control engineers are bound to use the conventional performance criteria. In this work, an LPV tower fore-aft damping controller is designed that is scheduled on the support structure frequency. The controller is designed to minimize the $\mathcal{H}_{2}$ norm of the closed-loop system (i.e., the energy sum of transients) from the wind input to a certain imposed performance output (here, the weighted sum of the tower top fore-aft acceleration and collective pitch angle control action) and the performance is finally evaluated in terms of fatigue loading on the tower.

Linear parameter-varying control and/or identification are not new in the field of wind energy. ${ }^{15-19}$ LPV control has been proposed for variable-speed fixed-pitch wind turbines in one study ${ }^{15}$ for controlling the rotor speed (RS) in both partial load and full load. This work is later extended in one study ${ }^{16}$ to variable-pitch wind turbines, where an LPV controller is developed scheduled by the wind speed, RS and pitch angle. Similar work is presented in one study; ${ }^{20}$ however, a more pragmatic approach is chosen wherein the scheduling parameter is the RS at below-rated wind conditions and the blade pitch angle at above-rated wind conditions. In two studies, ${ }^{21,22}$ the scheduling parameter is the estimated wind speed. LPV controllers have also been used for loads reduction, where the majority of the work considers above-rated wind conditions. ${ }^{23-25}$ In an attempt to improve on the standard industrial approach to designing wind turbine controllers where each control loop is designed individually, a very enthusiastic approach is proposed in a study ${ }^{17}$ that considers multiple-input multiple-output LPV control design with multiple performance objectives, namely RS control, drivetrain damping and tower fore-aft damping. The LPV controller covers both below-rated and above-rated wind conditions and is scheduled on the (estimated) wind speed.

What is common to the publications mentioned previously is that LPV control is applied to deal with changes in the underlying wind turbine model because of wind speed variations. None of aforementioned works, however, touches upon the problem considered in this paper, namely LPV control for tower fore-aft damping when a parameter of the model (the support structure frequency) deviates from its design value and/or changes in time because of design and operational uncertainties.

The paper is organized as follows. In Section 2, a wind turbine model is presented as used for control design and evaluation. The proposed approach to LPV tower fore-aft damping control is described in Section 3, in comparison with a traditional tower fore-aft damping control approach, and is further evaluated in Section 4 on a case study with the generic 5 MW NREL reference wind turbine. Finally, concluding remarks on the potential of the proposed method for reduction of the cost of offshore wind energy are made in Section 5.

\section{WIND TURBINE MODEL}

In this section, a wind turbine modeling approach that can be used towards addressing the formulated goals of the research work is given. This approach is applicable to variable-speed pitch-regulated horizontal-axis wind turbines with any number of blades and is used within industrial control software design tools for the development of control loops such as e.g. power regulation and RS control, drivetrain damping control, tower damping control, individual pitch control and rotor overspeed prevention control.

For control systems design, the higher-fidelity models that are typically used for wind turbine simulation are replaced by more simple models which represent the turbine dynamic behavior up to some extent relevant for the control purposes. ${ }^{1}$ 
Because of that the main subsystems of a wind turbine physically interact with each other, a natural modeling approach is to characterize this dynamic behavior of each subsystem individually, as shown in the remainder of this section.

\subsection{Aerodynamic modeling}

For a given wind turbine rotor with radius $R$ and constant uniform undisturbed wind speed $v$, let us define the dynamic force $F_{d y n}(v)=\frac{1}{2} \rho \pi R^{2} v^{2}$ with $\rho$ representing the air density. Assuming that there is one axial wind speed per turbine blade $u_{a x}^{(i)}$ for $i=\overline{1, B}$ where $B$ represents the number of turbine blades, the following blade-effective axial force $F_{a x}^{(i)}$ and aerodynamic torque $T_{a}^{(i)}$ can be defined, respectively:

$$
\begin{aligned}
& F_{a x}^{(i)}\left(u_{a x}^{(i)}\right)=F_{d y n}\left(u_{a x}^{(i)}\right) \cdot C_{T}\left(\lambda^{i}, \theta^{(i)}\right) / B \\
& T_{a}^{(i)}\left(u_{a x}^{(i)}\right)=R \cdot F_{d y n}\left(u_{a x}^{(i)}\right) \cdot C_{Q}\left(\lambda^{(i)}, \theta^{(i)}\right) / B
\end{aligned}
$$

appropriately catering for the effect of individual blade pitching. ${ }^{26}$ The static rotor thrust coefficient $C_{Q}$ and torque coefficient $C_{T}$ depend on the individual blade pitch angles $\theta^{(i)}$ and blade tip-speed ratios $\lambda^{(i)}=\frac{\Omega_{r} r}{u_{a x}^{(i)}}$ where $\Omega_{r}$ represents the RS.

Dynamic inflow i.e., the effect of dynamic wake adaptation as a result of an abrupt change of the blade pitch angle and/or the wind speed ${ }^{1}$ can be modeled within our context in a simplified fashion by approximating the effect of pitch angle variations on the forces and torques in equation (1) as follows: ${ }^{27}$

(1) pre-filtering the collective pitch angle $\theta_{c o l}=\sum_{i=1}^{B} \theta^{(i)}$ using two lead-lag filters with coefficients depending on the mean wind speed, giving filtered collective blade pitch angles $\theta_{C_{T}}$ and $\theta_{C_{Q}}$, respectively;

(2) computing the filtered blade pitch angles:

$$
\begin{aligned}
& \theta_{C_{T}}^{(i)}=\theta_{C_{T}}+\theta^{(i)}-\theta_{c o l} \\
& \theta_{C_{Q}}^{(i)}=\theta_{C_{Q}}+\theta^{(i)}-\theta_{c o l}
\end{aligned}
$$

(3) finally, replacing the blade pitch angles $\theta^{(i)}$ in (1) with the filtered versions from the expressions (2).

By further assuming that the blades are designed with equal aerodynamic efficiency along the blade radius, the leadwise force is constant whereas the flapwise force increases linearly with the radial position ${ }^{28}$ and the following expressions hold for the blade flap moments $M_{f l}^{(i)}$, blade tilt moment $M_{\text {tilt }}^{(i)}$, blade yaw moments $M_{\text {yaw }}^{(i)}$ and blade sideward force $F_{s d}^{(i)}$, respectively:

$$
\begin{aligned}
M_{f l}^{(i)} & =-\frac{2}{3} \cdot R \cdot F_{a x}^{(i)} \\
M_{\text {tilt }}^{(i)} & =\frac{2}{3} \cdot R \cdot F_{a x}^{(i)} \cdot \sin \left(\psi^{(i)}\right) \\
M_{y a w}^{(i)} & =-\frac{2}{3} \cdot R \cdot F_{a x}^{(i)} \cdot \cos \left(\psi^{(i)}\right) \\
F_{s d}^{(i)} & =-\frac{2}{R} \cdot T_{a}^{(i)} \cdot \sin \left(\psi^{(i)}\right)
\end{aligned}
$$

Note that all forces and moments have their directions according to a right-handed coordinate system with the $x$-axis pointing downwind and the $z$-axis pointing downwards. Additionally, $\psi^{(i)}$ denotes the azimuthal position of the $i^{\text {th }}$ blade (by convention, zero at 3 o'clock and increasing in clockwise direction).

\subsection{Drivetrain model}

Whereas the slow shaft of the drivetrain is flexible and modeled by the first collective lead-lag mode with torsional stiffness $s_{d t}$ and damping $d_{d t}$, the fast shaft is assumed rigid. The gearbox is rigidly connected to the rigid nacelle and has a transmission ratio of $i_{t r}$ which is conventionally positive when the direction of rotation of the fast shaft is the same as the one of the slow shaft and negative otherwise. The rotational dynamics of the two shafts are then represented by the following equations of motion: 


$$
\begin{aligned}
J_{g} \dot{\Omega}_{g} & =\frac{1}{i_{t r}} \cdot\left(T_{s h}-T_{\text {loss }}\right)-T_{\text {gen }} \cdot \operatorname{sign}\left(i_{\text {tr }}\right) \\
J_{r} \dot{\Omega}_{r} & =T_{a}-T_{s h}
\end{aligned}
$$

where $J_{r}$ and $J_{g}$ represent the rotor inertia and generator inertia, respectively, $\Omega_{g}$ is the generator speed, $T_{g e n}$ is the generator torque and $T_{a}$ is the rotor-effective aerodynamic torque, calculated similarly to blade-effective counterpart in equation (1) but using the collective wind speed defined in Subsection 2.5. The shaft torque $T_{s h}$ is given by the equation:

$$
T_{s h}=s_{d t} \gamma+d_{d t} \dot{\gamma}
$$

which depends on both the shaft torsion angle $\gamma$ and the shaft torsion velocity $\dot{\gamma}$ given by

$$
\dot{\gamma}=\Omega_{r}-\frac{1}{i_{t r}} \Omega_{g}
$$

The conversion losses can be modeled by:

$$
T_{\text {loss }}=T_{C}+T_{v} \frac{1}{i_{t r}} \Omega_{g}+T_{t} T_{s h}
$$

and arise because of three loss terms, namely the Coulomb damping torque $T_{C}$, the viscous friction torque $T_{v}$ and an additional term $T_{t}$ proportional to the shaft torque.

\subsection{Tower top motion}

The tower top motion can be modeled by a second-order mass-spring-damper system to approximate the first structural tower mode. The tower mass $m_{t}$, damping $d_{t}$ and stiffness $s_{t}$ are the parameters that fully describe this considered motion and can be considered to be the same both in the fore-aft direction and in the sidewards direction.

The fore-aft tower motion is excited by the axial forces $F_{a x}^{(i)}$ and the tilting moment $M_{\text {tilt }}^{(i)}$. By viewing, for the sake of simplicity, the tower as a cantilever prismatic beam, the tilting moment $M_{\text {tilt }}^{(i)}$ applied to the tower top will produce the same tower top displacement as a force $\frac{3}{2 H} M_{\text {till }}^{(i)}$, therefore resulting in the following fore-aft tower model:

$$
m_{t} \ddot{x}_{f a}+d_{t} \dot{x}_{f a}+s_{t} x_{f a}=\sum_{i=1}^{B}\left(F_{a x}^{(i)}-\frac{3}{2 H} M_{t i l t}^{(i)}\right)
$$

where $x_{f a}$ is the tower top position in the fore-aft direction. The negative sign in equation (8) is due to the fact that a positive tilting moment will give rise to a forward, and hence negative, tower top displacement.

A similar model holds for the motion with respect to the tower top sidewards position $x_{s d}$ :

$$
m_{t} \ddot{x}_{s d}+d_{t} \dot{x}_{s d}+s_{t} x_{s d}=\frac{3}{2 H} T_{n a c}+\sum_{i=1}^{B} F_{s d}^{(i)}
$$

This motion is excited by the sum of the sidewards forces $F_{s d}^{(i)}$ and by the effective torque $T_{n a c}$ :

$$
T_{\text {nac }}=T_{\text {gen }} \cdot \operatorname{sign}\left(i_{t r}\right)+T_{s h}-\frac{1}{i_{t r}} \cdot\left(T_{s h}-T_{\text {loss }}\right)
$$

acting on the top of the tower and called nacelle torque.

\subsection{Actuator dynamics}

For variable-speed pitch-regulated wind turbines, two actuation subsystems are available for turbine control, namely the electrical generator and the blade pitch actuators. The electrical generator is modeled by a second-order linear servomechanism:

$$
T_{g e n}=\frac{\omega_{g}^{2}}{s^{2}+2 \beta_{g} \omega_{g} s+\omega_{g}^{2}} \cdot T_{g e n}^{r e f}
$$

with $\omega_{g}$ as model cut-off frequency and $\beta_{g}$ as damping ratio. This actuator will provide an actual generator torque $T_{g e n}$ based on a torque demand $T_{g e n}^{r e f}$. 
The pitch actuator model for each blade consists of two parts: the first is a delayed second-order linear servomechanism with model cut-off frequency $\omega_{p t}$, damping ratio $\beta_{p t}$ and pure delay $\tau_{p t}$ :

$$
\theta_{\text {unlim }}^{(i)}=\frac{\omega_{p t}^{2}}{s^{2}+2 \beta_{p t} \omega_{p t} s+\omega_{p t}^{2}} \cdot e^{-\tau_{p t} s} \cdot \theta_{r e f}^{(i)}
$$

providing an unlimited pitch angle $\theta_{\text {unlim }}^{(i)}$ for each turbine blade based on a pitch demand $\theta_{\text {ref }}^{(i)}$; the second is a limitation component that restricts each unlimited blade pitch position $\theta_{\text {unlim }}^{(i)}$ and its associated speed $\dot{\theta}_{\text {unlim }}^{(i)}$ and acceleration $\ddot{\theta}_{\text {unlim }}^{(i)}$, giving rise to the corresponding limited pitch angles $\theta_{\text {lim }}^{(i)}$ through the limitation relations:

$$
\begin{aligned}
& \theta_{\min } \leq \theta_{\text {lim }}^{(i)} \leq \theta_{\max } \\
& \dot{\theta}_{\min } \leq \dot{\theta}_{\text {lim }}^{(i)} \leq \dot{\theta}_{\max } \\
& \ddot{\theta}_{\min } \leq \ddot{\theta}_{\text {lim }}^{(i)} \leq \ddot{\theta}_{\max }
\end{aligned}
$$

with defining constants $\left\{\theta_{\min }, \theta_{\max }\right\}$ for the blade pitch angle limitation, $\left\{\dot{\theta}_{\min }, \dot{\theta}_{\max }\right\}$ for the blade pitch speed limitation and $\left\{\ddot{\theta}_{\min }, \ddot{\theta}_{\max }\right\}$ for the blade pitch acceleration limitation.

\subsection{Wind modeling}

A simplified wind modeling approach based on the mentioned so-called blade-effective signals ${ }^{26,28}$ i.e. per blade there is one single wind signal that parametrizes the corresponding blade axial thrust force $F_{a x}^{(i)}$ and torque $T_{a}^{(i)}$ is used with the following effects included:

stochastic effects: $\quad$ blade-effective stochastic wind speed signals $u_{\text {sto }}^{(i)}$ for $i=\overline{1, B}$ are generated in such a way that they approximate the effect of a three-dimensional wind field on the spectrum of the flapwise blade root bending moments $M_{f l}^{(i)}$; these stochastic wind signals consist both of a slowly-varying average wind (so-called $0 P$ ) component and of periodic components with frequencies equal to multiples of the rotor rotation frequency $\frac{\Omega_{r}}{2 \pi}$ resulting in rotational wind field sampling (so-called $n P$ components with $n=1,2, \ldots$ );

deterministic effects: namely wind shear $u_{s h r}^{(i)}$, tower shadow $u_{\text {shd }}^{(i)}$ and the effect of fore-aft tower top motion $u_{\text {tow }}^{(i)}=$ $-\dot{x}_{f a} \cdot\left(1-\frac{R}{H} \cdot \sin \left(\psi^{(i)}\right)\right)$.

These are inputs to the aerodynamic model equation 1) through the defined blade-effective wind speeds $u_{a x}^{(i)}$ :

$$
u_{a x}^{(i)}=u_{\text {sto }}^{(i)}+u_{\text {shr }}^{(i)}\left(\psi^{(i)}\right)+u_{\text {shd }}^{(i)}\left(\psi^{(i)}\right)+u_{\text {tow }}^{(i)}\left(\psi^{(i)}\right)
$$

The collective wind speed $u_{c o l}$ introduced for the definition of the rotor-effective aerodynamic torque $T_{a}$ in equation (4) is defined as:

$$
u_{c o l}=\frac{1}{B} \sum_{i=1}^{B} u_{a x}^{(i)}
$$

and represents the average of the blade-effective wind speed signals.

\section{TOWER DAMPING CONTROL}

A wind turbine controller consists of an interconnection of different control loops (Figure 1), such as loops for regulating the RS and power production, damping oscillations in the drivetrain, reducing tower fore-aft vibrations, mitigating blade loads by individual pitch control and so on. The focal point of this section is the problem of fore-aft tower damping control design when the tower frequency is not known exactly during the design and/or might change during turbine operation because of external influences. Generally speaking, the purpose of the tower fore-aft damping control loop is to achieve support structure fatigue load reduction by means of collective pitch control as shown in Figure 1. By modifying the collective pitch angle of the blades in response to the measurement of the tower top accelerations, the fatigue loads can be reduced. The tower bottom moment (TBM) is considered here as an indicator of the loads on the support structure. ${ }^{1}$ In practice, it is of special importance to have an estimate of how the mechanical loads in the tower relate to the support 


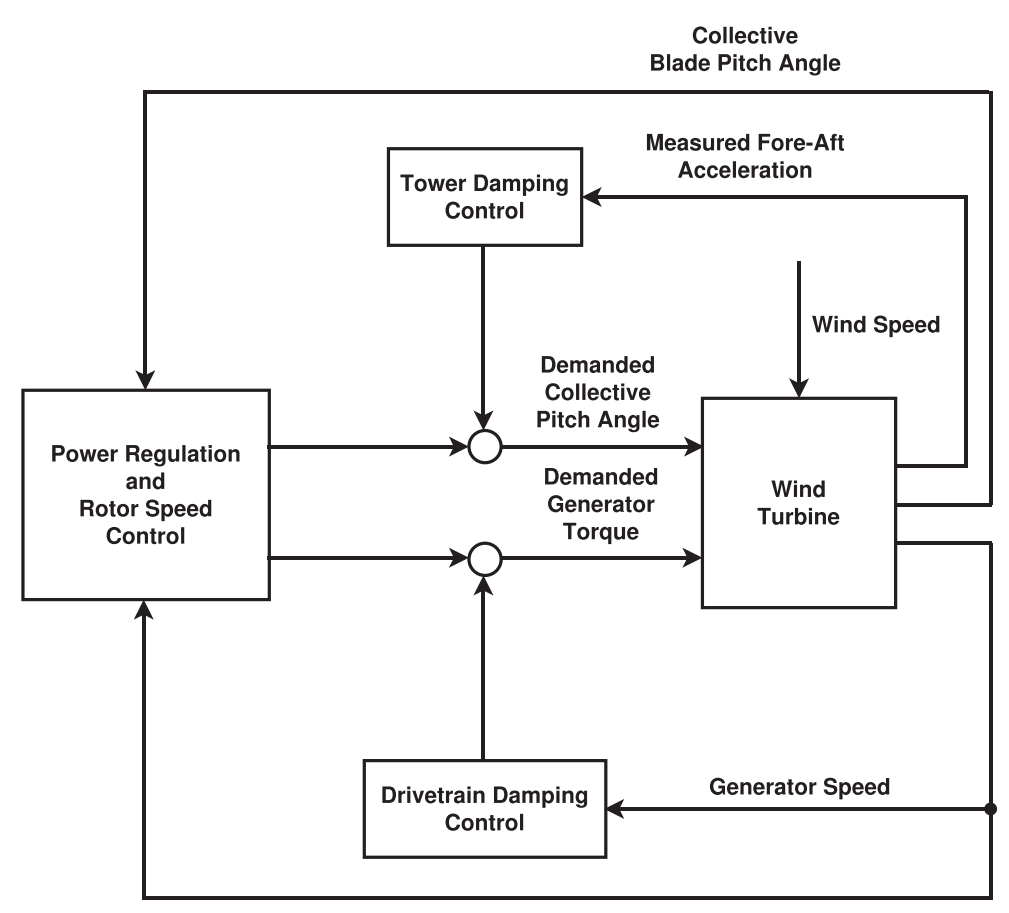

Figure 1. Simplified block diagram representation of the considered control loops.

structure lifetime. This is generally evaluated starting from the time series of the TBM and subsequently using this data within damage-equivalent load (DEL) calculation algorithms, such as the rainflow-counting algorithm, in order to arrive at an estimate of the equivalent fatigue load for the entire lifetime of the wind turbine. ${ }^{1}$ From a controls viewpoint, the problem of DEL load mitigation is not only challenging because of that the calculation of DELs can be done in many different ways, all yielding their separate results only correlated up to some extent, ${ }^{29}$ but also because of it is difficult to formulate control objectives (time-domain, frequency-domain or mixed) in terms of a desired DEL behavior for any given DEL estimation method since the involved estimation calculations are not straightforwardly representable by closed-form expressions. The problem of tower fatigue load reduction through active damping control is, therefore, always indirectly addressed through one or a combination of these formulated control objectives (for several previous works we refer to other studies ${ }^{10,30-34}$ ). An even more challenging problem arises when the support structure parameters used within the tower top motion model vary with the passing of time because of e.g., aging, ${ }^{1,5,6}$ scour, $^{4,7}$ marine sand dunes ${ }^{3,4}$ or biofouling. ${ }^{8}$ Although it is, in general, difficult to quantify the effects of such phenomena directly on the physical parameters $m_{t}, s_{t}, d_{t}$ of the model equation (8), practical experience of wind turbine manufacturers has revealed that these effects can be most easily translated in terms of variations of the first natural tower frequency $\omega_{n}=\sqrt{\frac{s_{t}}{m_{t}}}$ from its nominal value. It is, therefore, crucial for appropriate tower damping control operation that such changes in the first natural tower frequency are accounted for within the control action. It should be pointed out that the damping ratio is an even more significant source of model uncertainty than the frequency; however, uncertainty in the damping is not considered here because of its insignificant effect on the design and performance of wind turbine controller. The remainder of this section is dedicated to reviewing the nominal tower damping control approach in Subsection 3.1 where such variations are not explicitly accounted for and to presenting a state-of-the-art adaptive tower damping control approach in Subsection 3.2 where account of these variations is taken for properly adjusting the control action. These two approaches are then comparatively evaluated within a case study in Section 4.

\subsection{Nominal tower damping control}

For the design of a controller for fore-aft tower motion damping, the non-linear model presented in Section 2 is linearized in the vicinity $p$ of an equilibrium operating point $\bar{p} \triangleq\left[\theta_{\text {col }}\left(u_{\text {col,high }}\right) \Omega_{r}\left(u_{\text {col,high }}\right) u_{\text {col,high }}\right]^{T}$ in terms of some collective wind speed $u_{c o l, h i g h}$ for above-rated wind turbine operation, which gives rise to a collective pitch angle $\theta_{\text {col }}\left(u_{c o l, h i g h}\right)$ and RS $\Omega_{r}\left(u_{\text {col,high }}\right)$ based on the turbine operation curve.

The derived linearized model from Section 2 can be expressed by a state-space representation in terms of small deviations $\delta$ of all variables from their values at the considered operating point: 


$$
\begin{aligned}
& {\left[\begin{array}{l}
\delta \dot{x}_{f a} \\
\delta \dot{x}_{f a}
\end{array}\right]=\underbrace{\left[\begin{array}{cc}
0 & 1 \\
-\frac{s_{t}}{m_{t}} & -\frac{d_{t}+\nabla_{u_{c o l}} F_{a x}(\bar{p})\left(1+\frac{R^{2}}{2 H^{2}}\right)}{m_{t}}
\end{array}\right]}_{A_{f a}} \cdot\left[\begin{array}{l}
\delta x_{f a} \\
\delta \dot{x}_{f a}
\end{array}\right]+\underbrace{\left[\begin{array}{c}
0 \\
\frac{\nabla_{\theta_{c o l}} F_{a x}(\bar{p})}{m_{t}}
\end{array}\right]}_{B_{u, f a}} \delta \theta_{c o l}+\underbrace{\left[\begin{array}{c}
0 \\
\frac{\nabla_{u_{c o l}} F_{a x}(\bar{p})}{m_{t}}
\end{array}\right]}_{B_{w, f a}} \delta u_{s t o, c o l}} \\
& \delta \ddot{x}_{f a}=\underbrace{\left[-\frac{s_{t}}{m_{t}}-\frac{d_{t}+\nabla_{u_{c o l}} F_{a x}(\bar{p})\left(1+\frac{R^{2}}{2 H^{2}}\right)}{m_{t}}\right]}_{C_{f a}} \cdot\left[\begin{array}{l}
\delta x_{f a} \\
\delta \dot{x}_{f a}
\end{array}\right]+\underbrace{\frac{\nabla_{\theta_{c o l}} F_{a x}(\bar{p})}{m_{t}}}_{D_{u, f a}} \delta \theta_{c o l}+\underbrace{\frac{\nabla_{u_{c o l}} F_{a x}(\bar{p})}{m_{t}}}_{D_{w, f a}} \delta u_{s t o, c o l}
\end{aligned}
$$

with the derivatives of the aerodynamic thrust force $F_{a x}$ with respect to the collective pitch angle $\theta_{c o l}$ and collective wind speed $u_{c o l}$ at the equilibrium operating point $\bar{p}$ being computed as:

$$
\begin{aligned}
& \nabla_{\theta_{c o l}} F_{a x}(\bar{p})=\left.\frac{1}{2} \rho \pi R^{2} \bar{u}_{c o l}^{2} \frac{\partial C_{T}\left(\lambda, \theta_{c o l}\right)}{\partial \theta_{c o l}}\right|_{\bar{p}} \\
& \nabla_{u_{c o l}} F_{a x}(\bar{p})=\left.\frac{1}{2} \rho \pi R^{2} \bar{u}_{c o l} \frac{\partial C_{T}\left(\lambda, \theta_{c o l}\right)}{\partial u_{c o l}}\right|_{\bar{p}}
\end{aligned}
$$

Additionally, the term $\delta u_{\text {sto,col }}$ is introduced to account for both small deviations of the collective wind speed and small deviations of the tower top fore-aft velocity from the considered equilibrium point:

$$
\delta u_{s t o, c o l} \triangleq \delta u_{c o l}+\delta \dot{x}_{f a}
$$

Based on the state-space model (16), a controller can be designed. Here, a state-feedback type of proportional-derivative controller with respect to the deviations of the fore-aft acceleration $\ddot{x}_{f a}$ from an equilibrium is most easily designed by making use of the two states of the considered model; use is made of the linear quadratic regulator (LQR) design methodol$\mathrm{ogy}^{35}$ by an appropriate choice of a set $(Q, R)$ of state-weighting and input-weighting matrices. The collective pitch angle control signal is then expressed as:

$$
\delta \theta_{c o l}=\left[\begin{array}{ll}
K_{P} & K_{D}
\end{array}\right] \cdot\left[\begin{array}{l}
\delta \hat{x}_{f a} \\
\delta \hat{\dot{x}}_{f a}
\end{array}\right]
$$

and represents a linear combination of the estimated fore-aft tower top position $\delta \hat{x}_{f a}$ and speed $\delta \hat{\dot{x}}_{f a}$ deviations obtained through a state observer designed based on the model (16). These two states can be retrieved using e.g. a discrete-time Kalman filter ${ }^{36}$ constructed based on this model and fed with the measurements of the fore-aft tower top acceleration $\ddot{x}_{f a}$, see Kanev et al. ${ }^{37}$ for further details regarding the design of the tower state observer.

Because of that the design has been carried for a linearized model at one specific (above-rated) wind speed, to cover the entire wind turbine operation region, a gain-scheduling strategy can be applied to the control action; ${ }^{37}$ in general, the control action of the tower damping controller is reduced, or even eliminated, in partial-load conditions and this is accounted for in the gain-scheduling design. Additionally, it is crucial to pre-filter the computed collective pitch angle tower damping control action using band-stop filters before adding this signal to the loop of Figure 1, because the estimates $\delta \hat{x}_{f a}$ and $\delta \hat{\dot{x}}_{f a}$ are influenced by $B P, 2 B P$ and $3 B P$ components because of tower shadow and rotational sampling effects. Furthermore, the average values in these estimates due to e.g., bias in the measurement of $\ddot{x}_{f a}$ also need to be removed using a high-pass filter with cut-off frequency below the natural tower frequency. ${ }^{37}$ These filters, therefore, also become part of the tower damping controller. The presented nominal tower damping control loop can be observed in the standard block diagram of Figure 4.

\subsection{Adaptive tower damping control}

The tower damping control approach previously presented provides optimal fatigue load reduction if the model (16) considered for control design is time-invariant. As mentioned, this assumption is in practice violated because of slow changes in the turbine support structure parameters, often quantified in terms of the first natural tower frequency. By allowing for controller parameter adaptation to such changes, assumed to this extent to be triggered by variations in the fore-aft tower stiffness $s_{t}$, the performance of tower damping controllers can be guaranteed also in such off-spec situations.

To arrive at an affinely parameter-dependent LPV state-space model, define

$$
\xi \triangleq \frac{s_{t}}{m_{t}}
$$

which is directly related to the slowly-varying changing parameter $\omega_{n}$ by $\xi=\omega_{n}^{2}$. Equation (16) then takes the form: 


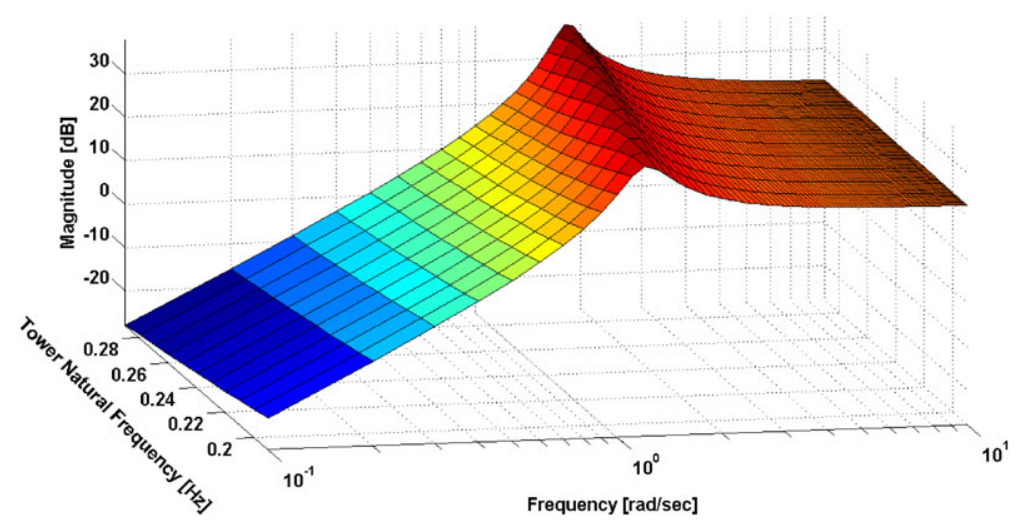

Figure 2. Bode plots for the $\delta \theta_{c o l} \rightarrow \delta \ddot{x}_{f a}$ transfer as a function of the tower natural frequency (directly related to $\xi$ by equation (20)).

$$
\begin{aligned}
{\left[\begin{array}{c}
\delta \dot{x}_{f a} \\
\delta \ddot{x}_{f a}
\end{array}\right] } & =A_{f a}(\xi) \cdot\left[\begin{array}{l}
\delta x_{f a} \\
\delta \dot{x}_{f a}
\end{array}\right]+B_{u, f a} \delta \theta_{c o l}+B_{w, f a} \delta u_{s t o, c o l} \\
\delta \ddot{x}_{f a} & =C_{f a}(\xi) \cdot\left[\begin{array}{l}
\delta x_{f a} \\
\delta \dot{x}_{f a}
\end{array}\right]+D_{u, f a} \delta \theta_{c o l}+D_{w, f a} \delta u_{s t o, c o l}
\end{aligned}
$$

with $A_{f a}(\xi)$ and $C_{f a}(\xi)$ depending affinely on $\xi$. In Figure 2, an example of the sensitivity of the $\delta \theta_{c o l} \rightarrow \delta \ddot{x}_{f a}$ transfer to the variation of $\xi$ is shown; as can be seen, the resonant mode of the system, typically damped by tower damping controllers, changes its location with changes in $\xi$.

Tower damping controllers whose parameters change based on the variation of the parameter $\xi$ can be synthesized within the framework of LPV control theory using linear matrix inequality (LMI) tools from convex optimization. ${ }^{38,39}$ The design problem will need, however, to be addressed through an output-feedback approach because the separation of state estimation and state-feedback control design cannot be directly tackled for LPV systems (see Friedland ${ }^{40}$ for further details). By first representing the model (21) as a discrete-time LPV system through appropriate discretization ${ }^{41}$ by e.g., zero-order hold transformation ${ }^{36}$ and appending all design specifications such as e.g., the LQR control synthesis weighting matrices $Q$ and $R$ to a so-called generalized plant:

$$
P:\left\{\begin{array}{c}
x_{P}(k+1)=A_{P}(\xi) x_{P}(k)+B_{P_{u}}(\xi) \delta \theta_{c o l}(k)+B_{P_{w}}(\xi) \delta u_{s t o, c o l}(k) \\
\delta \ddot{x}_{f a}(k)=C_{P_{y}}(\xi) x_{P}(k)+D_{P_{u y}}(\xi) \delta \theta_{c o l}(k)+D_{P_{w y}}(\xi) \delta u_{s t o, c o l}(k) \\
z(k)=C_{P_{z}}(\xi) x_{P}(k)+D_{P_{u z}}(\xi) \delta \theta_{c o l}(k)
\end{array}\right.
$$

a discrete-time controller:

$$
K:\left\{\begin{array}{c}
x_{K}(k+1)=A_{K}(\xi) x_{K}(k)+B_{K}(\xi) \delta \ddot{x}_{f a}(k) \\
\delta \theta_{c o l}(k)=C_{K}(\xi) x_{K}(k)+D_{K}(\xi) \delta \ddot{x}_{f a}(k)
\end{array}\right.
$$

can be designed by the LMI optimization approach for discrete-time $\mathcal{H}_{2}$ LPV output-feedback controller synthesis methodology using parameter-dependent Lyapunov functions, ${ }^{42-45}$ providing both stability and performance guarantees for the closed-loop system:

$$
T:\left\{\begin{array}{l}
x(k+1)=A(\xi) x(k)+B(\xi) \delta \ddot{x}_{f a}(k) \\
z(k)=C(\xi) x(k)
\end{array}\right.
$$

The output $z(k)$ in equations (22) and (24) represents the performance signal for the associated $\mathcal{H}_{2}$ optimization problem. In this study, it is selected as a weighted sum of the collective pitch angle $\delta \theta_{c o l}(k)$ and the fore-aft tower acceleration $\delta \ddot{x}_{f a}(k)$ as follows:

$$
z(k)=Q^{1 / 2} \cdot \delta \ddot{x}_{f a}(k)+R^{1 / 2} \cdot \delta \theta_{c o l}(k)
$$

wherein $Q^{1 / 2}$ and $R^{1 / 2}$ denote the (matrix) square roots of the corresponding components from the pair $(Q, R)$ used for the tuning of the state-feedback nominal tower damping controller by the LQR methodology (see e.g. Feron et al. ${ }^{46}$ for more details). In this way, minimizing the closed-loop transfer operator from the disturbance input to the performance output is similar to LQR optimization, with the difference that the controller developed here is an output-feedback controller 
as opposed to the previous state-feedback controller. The reason for using a cost function equal to the one used in LQR optimization is that choosing the same cost function allows one to make a fair comparison between the two controllers.

Before the main result for solving the $\mathcal{H}_{2}$ optimization problem for controller (23) design is reported, the following lemma is needed (see e.g., Feron et al. ${ }^{47}$ for more details).

Lemma 3.1. (Linear matrix inequality problem for discrete-time $\mathcal{H}_{2}$ linear parameter-varying output-feedback controller synthesis)

The closed-loop system (24) with generalized plant (22) and controller (23), parametrized in terms of the slowly-varying parameter $\xi \in \Xi$ with $\frac{d}{d t} \xi \approx 0$ is Lyapunov (quadratically) stable and satisfies the generalized $\mathcal{H}_{2}$ quadratic performance criterion $\|T\|_{2}<\sigma$ if the following optimization problem with LMI constraints is feasible in terms of the symmetric affinely parameter-dependent matrix $X(\xi)=X_{0}+X_{1} \xi$ and symmetric parameter-independent matrix $Q$ :

$$
\begin{aligned}
& \text { minimize } \sigma^{2} \\
& \text { over all } \xi \in \Xi
\end{aligned}
$$

subject to the constraints:

$$
\begin{aligned}
{\left[\begin{array}{ccc}
X(\xi) & X(\xi) A(\xi) & X(\xi) B(\xi) \\
\star & X(\xi) & 0 \\
\star & \star & I
\end{array}\right] } & \prec 0 \\
{\left[\begin{array}{cc}
Q & C(\xi) \\
\star & X(\xi)
\end{array}\right] } & \succ 0 \\
\operatorname{trace}(Q) & <\sigma^{2}
\end{aligned}
$$

Note, however, that the constraints in Lemma 3.1 are not convex in the unknowns because of products between the unknown matrix $X(\xi)$ and the closed-loop matrices of (24) which contain the unknown controller matrices. However, these can be further manipulated, leading to Lemma 3.2 which provides convex constraints for the LMI optimization problem in the new unknowns $R(\xi), S(\xi), K(\xi), L(\xi), M(\xi)$ and $N(\xi)$.

Lemma 3.2 (Linear matrix inequality problem with convex constraints for discrete-time $\mathcal{H}_{2}$ linear parameter-varying output-feedback controller synthesis). The closed-loop system (24) with generalized plant (22) and controller (23), parametrized in terms of the slowly-varying parameter $\xi \in \Xi$ with $\frac{d}{d t} \xi \approx 0$ is Lyapunov (quadratically) stable and satisfies the generalized $\mathcal{H}_{2}$ quadratic performance criterion $\|T\|_{2}<\sigma$ if the following optimization problem with LMI constraints is feasible in terms of the symmetric affinely parameter-dependent matrices $R(\xi)=R_{0}+R_{1} \xi, S(\xi)=S_{0}+S_{1} \xi, K(\xi)=$ $K_{0}+K_{1} \xi, L(\xi)=L_{0}+L_{1} \xi, M(\xi)=M_{0}+M_{1} \xi, N(\xi)=N_{0}+N_{1} \xi$ and symmetric parameter-independent matrix $Q:$

$$
\begin{aligned}
& \text { minimize } \sigma^{2} \\
& \text { over all } \xi \in \Xi
\end{aligned}
$$

subject to the constraints:

$$
\begin{aligned}
& {\left[\begin{array}{ccccc}
S(\xi) & I & A_{P}(\xi) S(\xi)+B_{P_{u}}(\xi) M(\xi) & A_{P}(\xi)+B_{P_{u}}(\xi) N(\xi) C_{P_{y}}(\xi) & B_{P_{w}}(\xi)+B_{P_{u}}(\xi) N(\xi) D_{P_{w y}}(\xi) \\
\star & R(\xi) & K(\xi) & S(\xi) A_{P}(\xi)+L(\xi) C_{P_{y}}(\xi) & S(\xi) B_{P_{w}}(\xi)+L(\xi) D_{P_{w y}}(\xi) \\
\star & \star & S(\xi) & I & 0 \\
\star & \star & \star & R(\xi) & 0 \\
\star & \star & \star & \star & I
\end{array}\right] \prec 0} \\
& {\left[\begin{array}{ccc}
Q & C_{P_{z}}(\xi) S(\xi)+D_{P_{u z}}(\xi) M(\xi) & C_{P_{z}}(\xi)+D_{P_{u z}}(\xi) N(\xi) C_{P_{y}}(\xi) \\
\star & S(\xi) & I \\
\star & \star & R(\xi)
\end{array}\right]} \\
& <\sigma^{2}
\end{aligned}
$$

Proof. Denote $X(\xi)$ in equation (26) of Lemma 3.1 together with $X^{-1}(\xi)$ and $Y(\xi)$ as

$$
X(\xi) \triangleq\left[\begin{array}{cc}
R(\xi) & U(\xi) \\
U^{T}(\xi) & Z(\xi)
\end{array}\right], X^{-1}(\xi) \triangleq\left[\begin{array}{cc}
S(\xi) & V(\xi) \\
V^{T}(\xi) & W(\xi)
\end{array}\right] \text { and } Y(\xi) \triangleq\left[\begin{array}{cc}
S(\xi) & I \\
V^{T}(\xi) & 0
\end{array}\right]
$$


Then the following change of variables becomes suitable:

$$
\left[\begin{array}{cc}
K(\xi) & L(\xi) \\
M(\xi) & N(\xi)
\end{array}\right]=\left[\begin{array}{cc}
U(\xi) & R(\xi) B_{P_{u}}(\xi) \\
0 & I
\end{array}\right]\left[\begin{array}{cc}
A_{K}(\xi) & B_{K}(\xi) \\
C_{K}(\xi) & D_{K}(\xi)
\end{array}\right]\left[\begin{array}{cc}
V^{T}(\xi) & 0 \\
C_{P_{y}}(\xi) S(\xi) & I
\end{array}\right]+\left[\begin{array}{cc}
R(\xi) A_{P}(\xi) S(\xi) & 0 \\
0 & 0
\end{array}\right]
$$

for rendering the constraints (26) convex by left-hand and right-hand size multiplication of the first LMI in equation (26) by $\operatorname{diag}\left\{Y^{T}(\xi), Y^{T}(\xi), Y^{T}(\xi)\right\}$ and $\operatorname{diag}\{Y(\xi), Y(\xi), Y(\xi)\}$, respectively, and of the second LMI in equation (26) by $\operatorname{diag}\left\{I, Y^{T}(\xi)\right\}$ and $\operatorname{diag}\{I, Y(\xi)\}$, respectively. Then the new constraints (27) of Lemma 3.2 arise and are convex in the unknowns $R(\xi), S(\xi), K(\xi), L(\xi), M(\xi), N(\xi)$ and $Q$.

For computational implementation of the LMI optimization problem of Lemma 3.2 towards controller synthesis, an approach based on gridding the parameter space $\Xi$ is used as in one study ${ }^{48}$ since the constraints (27) are not affine in the parameter $\xi$. A summary of the procedure for design and implementation of the LPV tower fore-aft damping controller is provided as follows:

Design stage (offline)

Step 1: define $\xi$ as in equation (20) and choose a polytopic range for $\xi \in \Xi$;

Step 2: choose a sufficiently fine grid of points for the parameter space $\Xi$;

Step 3: for each value of $\xi$ from the grid form the state-space system matrices (22);

Step 4: for all defined grid points solve optimization problem of Lemma 3.2 (using e.g. ${ }^{49}$ ) to obtain the matrices $R(\xi), S(\xi), K(\xi), L(\xi), M(\xi)$ and $N(\xi)$.

Implementation stage (online)

Step 5: at discrete real-time instant $k$, given estimate of $\xi$ of (20), form the current affine parameter-dependent system matrices (22);

Step 6: form the current affine parameter-dependent synthesis matrices $R(\xi), S(\xi), K(\xi), L(\xi), M(\xi)$ and $N(\xi)$;

Step 7: $\quad$ calculate $U(\xi)=I-R(\xi) S(\xi)$ and $V^{T}(\xi)=I$;

Step 8: use the reverse substitution (30) below to find the current controller matrices.

$$
\begin{aligned}
& D_{K}(\xi)=N(\xi) \\
& C_{K}(\xi)=\left(M(\xi)-D_{K}(\xi) C_{P_{y}}(\xi) S(\xi)\right) V^{-T}(\xi) \\
& B_{K}(\xi)=U^{-1}(\xi)\left(L(\xi)-R(\xi) B_{P_{u}}(\xi) D_{K}(\xi)\right) \\
& A_{K}(\xi)=U^{-1}(\xi)\left[\left(K(\xi)-L(\xi) C_{P_{y}}(\xi) S(\xi)-R(\xi) A_{P}(\xi) S(\xi)\right) V^{-T}(\xi)-R(\xi) B_{P_{u}}(\xi) C_{K}(\xi)\right]
\end{aligned}
$$

Observe in the procedure summarized previously that the most involved calculations related to solving the LMI optimization are performed offline to prepare the necessary data for online adaptation. The online controller adaptation, by itself, consists of matrix calculations of relatively low complexity. Therefore, the controller structure is not seen as restrictive from the implementation point of view. The design process proposed, however, is more involved than the conventional approach, and more simplified design approaches are also conceivable. However, the proposed LPV design approach offers important advantages such as guaranteed stability and performance for the complete range of parameter uncertainty, smooth variation of the controller parameters and does not rely on switching between local linear time-invariant controllers that do not share the same physical state.

In Figure 3, one example of the $\delta \ddot{x}_{f a} \rightarrow \delta \theta_{c o l}$ transfer, that results by using this synthesis procedure on a specific wind turbine using in this study, is shown; notice the change of the controller characteristics as a function of $\xi$. In Figure 4 , a schematic representation of the tower damping control is shown. The nominal tower damping control loop contains, as mentioned, an acceleration sensor that measures the fore-aft tower top position and based on it computes a tower damping control action by means of collective pitch control; the control action is pre-filtered and then fed to the blade pitch actuators. In situations where the turbine parameters such as the natural tower frequency differ from their nominal values used for the design of the tower damping controller, the loop will not perform as expected and fatigue load reduction will be limited. By contrast, the use of an adaptation mechanism as shown in Figure 4 could allow this control loop to react to changes in the natural tower frequency by adjusting the controller parameters accordingly. When used in conjunction with an online algorithm for estimation of the tower frequency, the controller update will be performed only occasionally when the tower frequency estimate has changed significantly, e.g., at a time scale of days to weeks. The estimation of the tower frequency falls outside the scope of this paper as this poses no technological challenge. Notice that besides its use for such online 


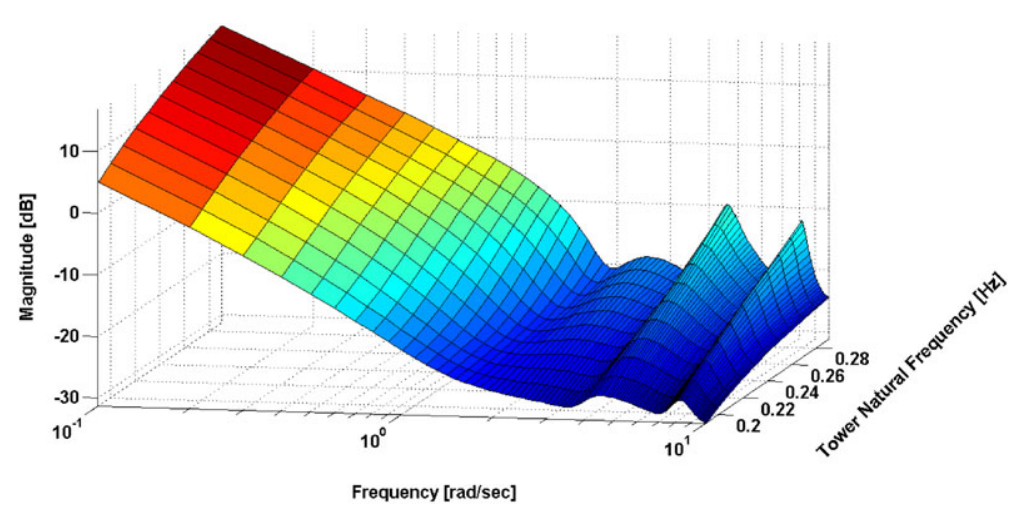

Figure 3. Bode plots for the $\delta \ddot{x}_{f a} \rightarrow \delta \theta_{c o l}$ transfer of the LPV controller scheduled on $\xi$ via the tower natural frequency.

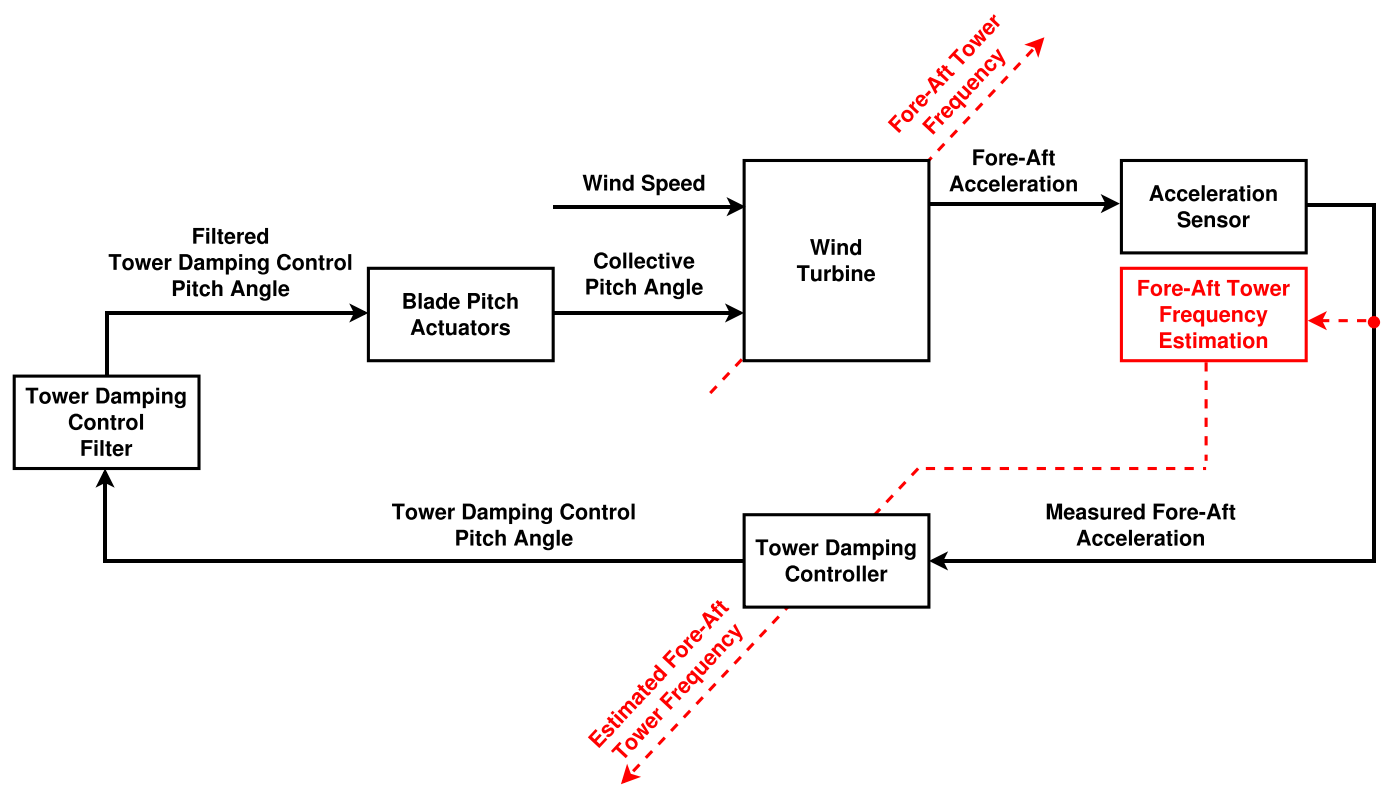

Figure 4. Conceptual view of a nominal tower damping control loop (in black) with the addition of an adaptation mechanism based on the estimated fore-aft tower frequency(in red).

adaptation, the presented adaptive controller could also be used to enable simplified manual fine-tuning, for instance, after turbine installation, so as to ensure optimized operation for the actual measured tower frequency.

It should also be pointed out that, in addition to the adaptive controller (23), similarly, the same notch filters are used as in the conventional tower damping controller (Figure 3).

\section{CASE STUDY}

In this section, a comparison of two tower damping control approaches as presented in Section 3 is given in terms of fatigue load reduction performance. The study is focused on investigating the DEL mitigation potential of these two strategies whenever the first fore-aft natural tower frequency is different from some nominal value.

Within the case study use is made of a generic three-bladed variable-speed pitch-regulated 5 MW bottom-supported monopile offshore horizontal-axis ${ }^{50}$ based on a concept design. ${ }^{51}$ The following choices have been made in this case study:

Considered range for adaptive tower damping control design: a realistic range $\Xi$ for the scheduling parameter $\xi$ has been chosen such that $\omega_{n} \in\left[\omega_{n, n o m}-20 \%, \omega_{n, n o m}+20 \%\right]$; the simulations and analysis are performed for five values of $\omega_{n}$ within this interval; 


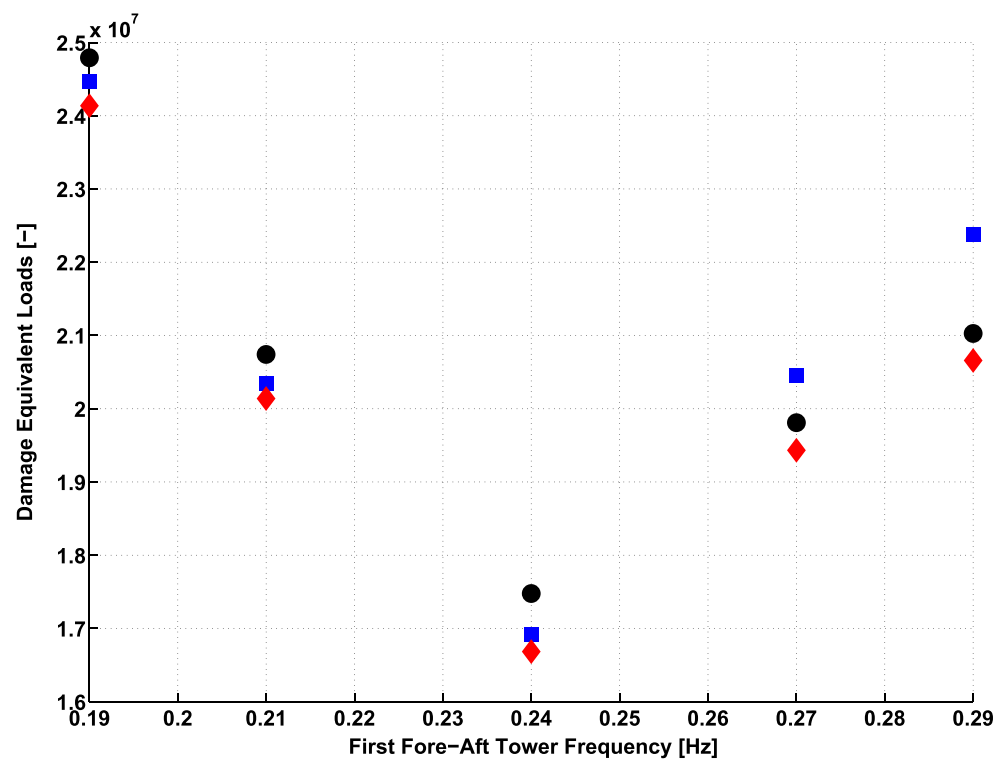

Figure 5. Obtained damage-equivalent loads for partial-load conditions: with tower damping control disabled (), with nominal tower damping control $(\square)$ and with adaptive tower damping control $(\boldsymbol{\nabla})$.

Table I. Main turbine operation data for a single worst-case DELs simulation scenario in partial-load conditions.

\begin{tabular}{lcc}
\hline & Nominal tower damping control & Adaptive tower damping control \\
\hline $\operatorname{MAX}\left(\Omega_{r}\right)[\mathrm{rpm}]$ & 11.49 & 11.48 \\
$\mathrm{SD}\left(\Omega_{r}\right)[\mathrm{rpm}]$ & 1.46 & 1.46 \\
$\mathrm{SD}\left(\dot{\theta}_{\mathrm{col}}\right)\left[{ }^{\circ} / \mathrm{s}\right]$ & 0.44 & 0.75 \\
Energy $[\mathrm{MWh}]$ & 6.13 & 6.12 \\
Tower DELs [-] & $2.49 \cdot 10^{7}$ & $2.49 \cdot 10^{7}$ \\
\hline
\end{tabular}

$\mathrm{DEL}$, damage-equivalent load; SD, standard deviation.

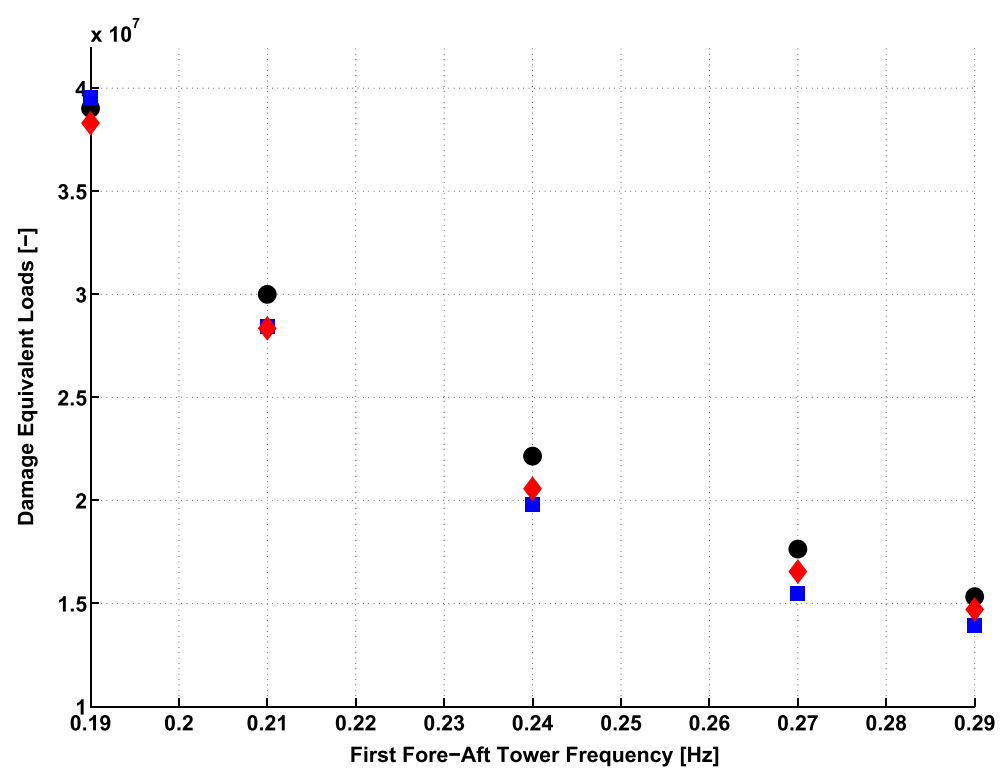

Figure 6. Obtained DELs for full-load conditions: with tower damping control disabled $(\mathbf{0})$, with nominal tower damping control ( and with adaptive tower damping control $(\boldsymbol{\vee})$. 
Table II. Main turbine operation data for a single worst-case DELs simulation scenario in full-load conditions.

\begin{tabular}{lcc}
\hline & Nominal tower damping control & Adaptive tower damping control \\
\hline $\operatorname{MAX}\left(\Omega_{r}\right)[\mathrm{rpm}]$ & 13.07 & 13.18 \\
$\mathrm{SD}\left(\Omega_{r}\right)[\mathrm{rpm}]$ & 0.41 & 0.42 \\
$\mathrm{SD}\left(\dot{\theta}_{c o l}\right)[\% / \mathrm{s}]$ & 0.91 & 1.26 \\
Energy $[\mathrm{MWh}]$ & 41.5 & 41.5 \\
Tower DELs [-] & $3.40 \cdot 10^{7}$ & $3.13 \cdot 10^{7}$ \\
\hline
\end{tabular}

$\mathrm{DEL}$, damage-equivalent load; SD, standard deviation.
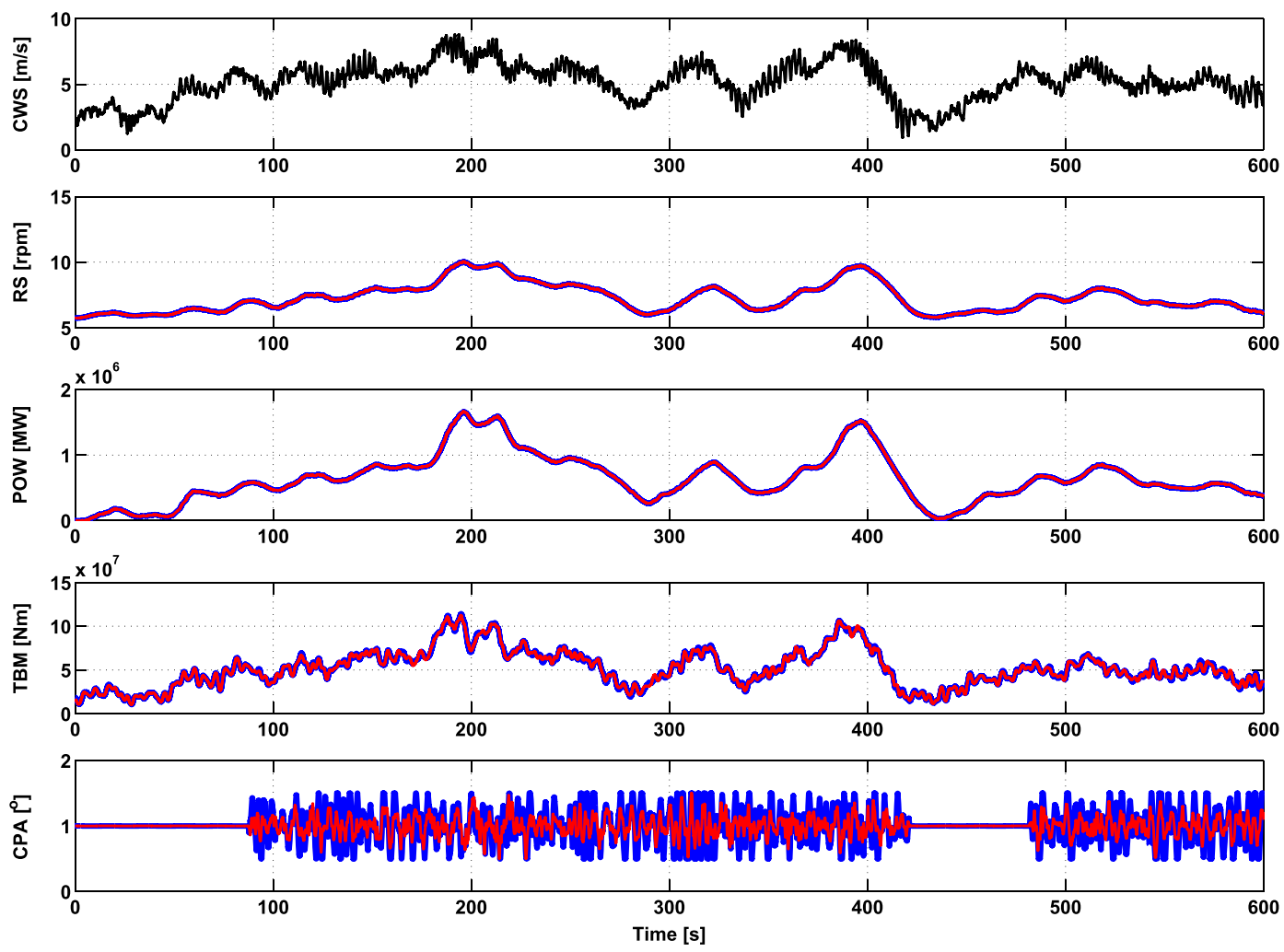

Figure 7. Time series for a single worst-case damage-equivalent loads simulation scenario in partial-load conditions: simulation wind speed (_ $)$, simulation with nominal tower damping control ) and simulation with adaptive tower damping control (

Tower damping control design conditions: the tower damping controller is designed at $20 \mathrm{~m} / \mathrm{s}$ and is further not scheduled on the wind speed;

Wind realization: two mean wind speeds are considered, one in partial-load conditions $(5.25[\mathrm{~m} / \mathrm{s}])$ and one in full-load conditions $(20[\mathrm{~m} / \mathrm{s}])$. For each mean wind speed, three wind realizations (wind seeds) are generated and simulated to enable an accurate estimate of the DELs;

Fatigue load calculation: the overall DELs are calculated using the rainflow-counting algorithm, separately for partial-load conditions and full-load conditions as an average of the DELs corresponding to each of the three wind realizations for a given value of the investigation parameter $\xi$;

Simulation duration: the simulations have been run for $650 \mathrm{~s}$ (data from the first $50 \mathrm{~s}$ discarded to avoid that initial transients affect the results).

The tower damping control loop can either be setup as nominal tower damping control, where the control strategy described in Subsection 3.1 is tuned based on the nominal first fore-aft natural tower frequency or as adaptive tower damping control, where the control strategy described in Subsection 3.2 is applied and the controller is fine-tuned based on the parameter $\xi$ that relates to the first fore-aft tower frequency used for the simulation model. An additional inves- 

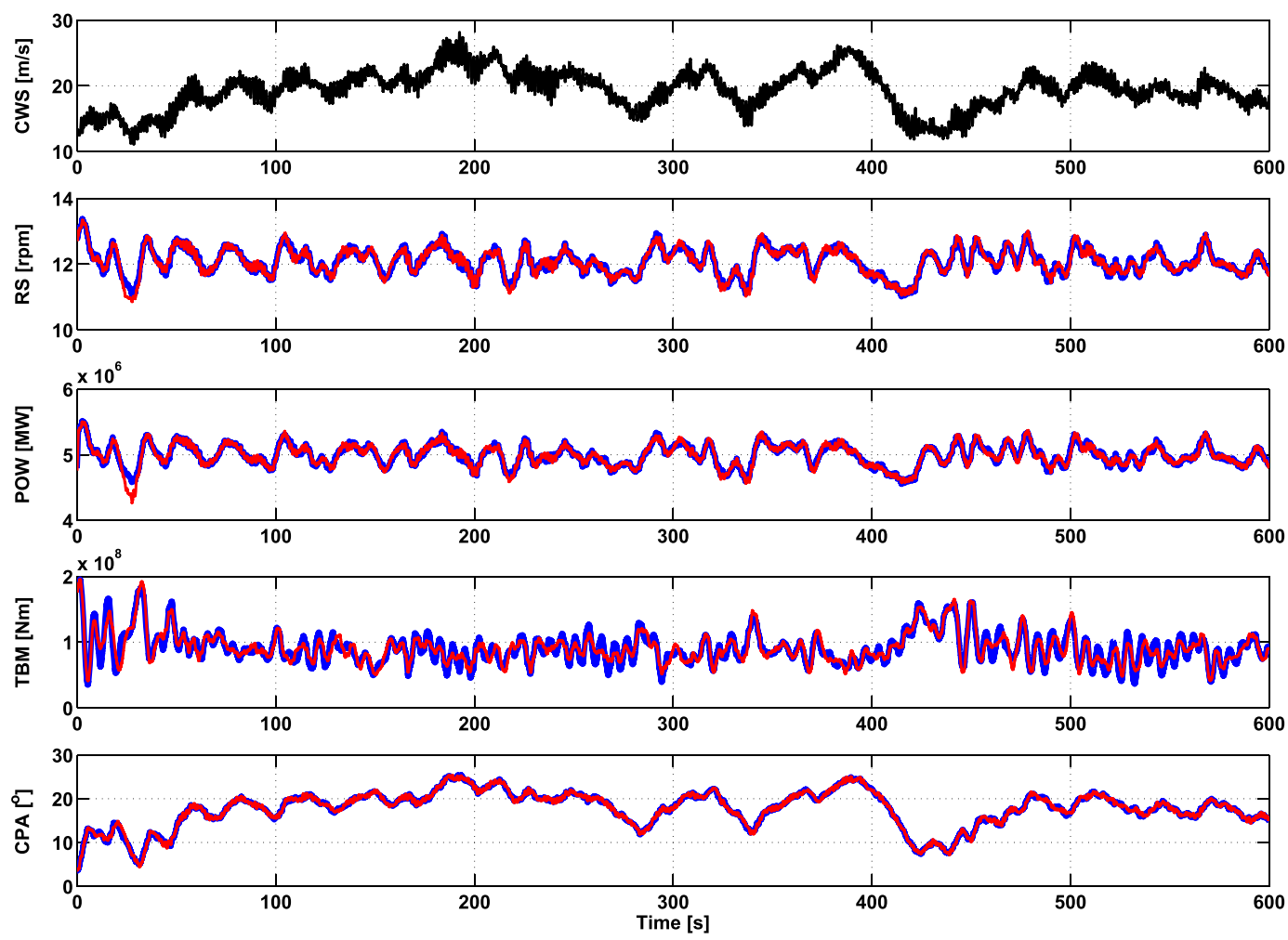

Figure 8. Time series for a single worst-case damage-equivalent loads simulation scenario in full-load conditions: simulation wind

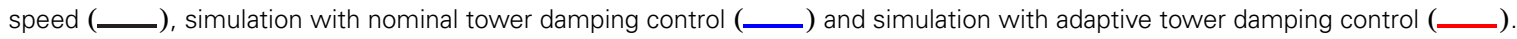

tigation setting called tower damping control disabled serves to give an indication of the open-loop fatigue loads on the turbine tower for the different simulation scenarios. The remainder of this section is dedicated to the analysis of the simulation results.

In Figure 5, the DELs for partial-load conditions are displayed for the range of investigated fore-aft tower frequencies. As can be seen, both nominal tower damping control and adaptive tower damping control allow for fatigue load reduction when the first fore-aft tower natural frequency is below its nominal value; however, the latter allows for better performance in terms of DELs and reduces by up to $1.5 \%$, the worst-case fatigue loads that would be typically obtained by nominal tower damping control. On the other hand, it can be observed that whenever the first fore-aft tower natural frequency is above its nominal value, the adaptive tower damping control approach still performs well with respect to the loads obtained by tower damping control disabled, whereas the nominal tower damping control approach does not and, on the contrary, will even increase the fatigue loads when compared with the open-loop loads by up to $6.5 \%$. Inspection of the associated wind turbine operation data for one single worst-case support structure DEL simulation, given in Table I, reveals that the added benefit of the adaptive tower damping controller is, as expected, small for partial-load conditions: though the RS regulation remains almost unaffected, as can be noticed by the similar values of the maximimum RS (MAX $\left(\Omega_{r}\right)$ ) and its standard deviation (SD $\left(\Omega_{r}\right)$ ), the collective blade pitch activity is increased according to the SD of the collective pitch rate $\left(\mathrm{SD}\left(\dot{\theta}_{c o l}\right)\right)$; finally, the energy production (Energy) and the support structure DELs (Tower DELs) are almost the same when comparing the nominal tower damping control approach to the adaptive tower damping control one (note that these values are different from the ones that can be observed in Figure 5 because these are the results corresponding to only one wind seed, whereas Figure 5 shows the mean DELs for three different wind seeds).

In terms of full-load performance, as shown in Figure 6, the adaptive tower damping control approach performs well for the entire range of investigated fore-aft natural tower frequencies and reduces the fatigue loads by up to $7 \%$ compared with the open-loop fatigue loads. As a general trend, for natural tower frequencies above the nominal value, this control strategy is outperformed by the nominal tower damping. However, when the natural tower frequency drops below its nominal value, the latter approach is seen yet again to be less advantageous as DEL reduction becomes less pronounced and, in the worst-case situation, the obtained DELs can even be higher than the open-loop fatigue loads. For this mentioned situation, on the other hand, the adaptive tower damping control strategy does reduce both the loads obtained with tower damping control disabled and the ones obtained with nominal tower damping control by as much as $3 \%$. Although this 
may seem little, for wind turbine manufacturers, this does translate to an approximate saving of $3 \%$ on the costs of the support structure!

The wind turbine operation data for a single worst-case support structure DEL simulation, displayed in Table II, shows that the added benefit of the LPV tower damping control approach is here validated in terms of the wind turbine performance: the RS regulation remains almost unaffected, while the collective blade pitching activity is slightly increased (notice by inspection of the SD of the collective blace pitch rates that a daptive tower damping control is not significantly more aggressive than nominal tower damping control). The energy production is almost the same for the two approaches, whereas the resulting of the support structure DELs are significantly lower with the adaptive tower damping controller.

The performance of the investigated approaches can also be seen in the light of the obtained simulation time series for e.g., the simulation scenarios corresponding to the worst-case DELs noticed. In Figure 7, it can be observed that for below-rated collective wind speeds, the power performance of the implemented control system does not differ considerably between the cases when nominal tower damping control or the adaptive tower damping control is used. Power regulation and RS control performance is similar. However, the slight differences between the two time series of the TBM further show that for this scenario, where a relative DEL reduction of up to $1.5 \%$ had been obtained by adaptive tower damping control, this had been possible with limited collective pitch angle (CPA) activity. For the above-rated conditions of Figure 8, it can be seen that the corresponding proposed relative 3\% DEL reduction had been possible by reasonable pitching activity and slightly weaker power performance.

\section{CONCLUSIONS}

This paper has dealt with the problem of adapting wind turbine tower damping controller parameters according to changes in the turbine support structure properties, parametrized in terms of the first fore-aft natural tower frequency. The exposition has begun by the proposition of a wind turbine model that can be used for control design. Subsequently, attention has been paid to both explaining a current design approach for the development of the tower damping control loop and to proposing a new strategy that improves upon the current technology, allowing the controller to adapt to changes in the natural tower frequency. The design approach that enables this strategy has been rooted in the LPV control theory. The two approaches to turbine tower damping control have eventually been evaluated in terms of DEL reduction capability within a case study for a generic wind turbine.

The study has revealed that the current approach to tower damping control does not provide optimal fatigue load reduction performance when the natural tower frequency of the wind turbine is different from its nominal value, typically used for the design of the controller: whereas in some situations the DEL reduction is less significant than in the nominal case, several scenarios have shown that such an approach could even increase DELs when compared with open-loop operation. On the other hand, the proposed adaptive tower control strategy has been shown to bring guaranteed and satisfactory performance in almost all situations. This strategy allows for a reduction of fatigue loads on the support structure of up to $3 \%$, which indicates that a reduction of the support structure costs of the same order can be expected by using the proposed adaptive control algorithm.

\section{ACKNOWLEDGEMENTS}

The financial support of TKI Wind op Zee for the Design for Reliable Power Performance (D4REL) project, part of which this work has been performed, is acknowledged. The first author would like to thank Prof. Roland Tóth from Technische Universiteit Eindhoven for discussions regarding the discretization of LPV systems.

\section{REFERENCES}

1. Burton T, Sharpe D, Jenkins N, Bossanyi E. Wind Energy Handbook. John Wiley \& Sons Ltd: Chichester, U.K, 2001.

2. Manwell JF, McGowan JG, Rogers AL. Wind Energy Explained: Theory, Design and Application. John Wiley and Sons Ltd: West Sussex, England, 2009.

3. Damgaard M, Andersen JKF, Ibsen LB, Andersen LV. Natural frequency and damping estimation of an offshore wind turbine structure. Proceedings of 22nd, International Offshore and Polar Engineering Conference, Rhodes, Greece, 2012; 300-307.

4. Damgaard M, Andersen JKF, Ibsen LB, Andersen LV. Time-varying dynamic properties of offshore wind turbines evaluated by modal testing. Proceedings of 18th, International Conference on Soil Mechanics and Geotechnical Engineering, Paris, France, 2013; 93-97. 
5. Fischer T, de Vries W, Rainey P, Schimdt B, Argyriadis K, Kühn M. Offshore support structure optimization by means of integrated design and controls. Wind Energy 2012; 15(1): 99-117.

6. Rendon Erica A, Manuel L. Long-term loads for a monopile-supported offshore wind turbine. Wind Energy 2014; 17(1): 209-223.

7. van der Tempel J, Zaaijer MB, Subroto H. The effects of scour on the design of offshore wind turbines. In Proceedings of the Third International Conference on Marine Renewable Energy, Blyth, United Kingdom, 2004; 53-62.

8. Wilson Jennifer C, Elliott M. The habitat-creation potential of offshore wind farms. Wind Energy 2009; 12(1): $203-212$.

9. Balaguer IF, Kanev S, Tcherniak D, Rossetti M. System identification methods on Alstom ECO 100 wind turbine. Proceedings of The Science of Making Torque from Wind (TORQUE 2010) Conference, Heraklion, Greece, 2010.

10. Fischer B, Shan M, A survey on control methods for the mitigation of tower loads, Technical Report (No. 01/104256), Fraunhofer IWES - Institute for Wind Energy and Energy Systems Technology, 2013.

11. Apkarian $P$, Gahinet $P$, Becker G. Self-scheduled $\left(\mathcal{H}_{\infty}\right)$ control of linear parameter-varying systems: a design example. Automatica 1995; 31(9): 1251-1261.

12. Apkarian P, Gahinet P. A convex characterization of gain-scheduled $\left(\mathcal{H}_{\infty}\right)$ controllers. IEEE Transactions on Automatic Control 1995; 40(5): 853-864.

13. Becker G, Packard A. Robust performance of linear parametrically-varying systems using parametrically- dependent linear feedback. Systems \& Control Letters 1994; 23: 205-215.

14. Skogestad S, Postlethwaite I. Multivariable Feedback Control: Analysis and Design. John Wiley and Sons: Chichester, U.K, 2005.

15. Bianchi FD, Mantz RJ, Christiansen CF. Control of variable-speed wind turbines by LPV gain scheduling. Wind Energy 2004; 10(7): 1-8.

16. Bianchi FD, De Battista H, Mantz RJ. Wind Turbine Control Systems: Principles, Modelling and Gain Scheduling Design. Springer-Verlag: London, U.K, 2007.

17. Østergaard KZ, Brath P, Stoustrup J. Linear Parameter-varying control of wind turbines covering both partial load and full load conditions. International Journal of Robust and Nonlinear Control 2009; 19(1): 92-116.

18. van Wingerden JW, Houtzager I, Felici F, Verhaegen M. Closed-loop identification of the time-varying dynamics of variable-speed wind turbines. Special Issue of International Journal of Robust and Nonlinear Control on Wind Turbines: New Challenges and Advanced Control Solutions 2009; 19(1): 4-21.

19. Gebraad PMO, van Wingerden JW, Fleming PA, Wright AD. LPV identification of wind turbine rotor vibrational dynamics using periodic disturbance basis functions. IEEE Transactions of Control Systems Technology 2013; 21(4): 1183-1190.

20. Lescher F, Zhao JY, Borne P. Robust gain-scheduling controller for pitch-regulated variable-speed wind turbine. Studies in Informatics and Control 2005; 14(4): 299-315.

21. Østergaard KZ, Brath P, Stoustrup J. Rate-bounded LPV control of a wind turbine in full load. In Proceedings of the IFAC World Congress, Seoul, Korea, 2008; 5593-5598.

22. Østergaard KZ, Brath P, Stoustrup J. Gain-scheduled linear quadratic control of wind turbines operating at high wind speed. In Proceedings of the IEEE Conference on Control Applications, Singapore, 2007; 276-281.

23. Lescher F, Zhao JY, Borne P. Switching LPV controllers for a variable-speed pitch-regulated wind-turbine. International Journal of Computers, Communications and Control 2006; 1(4): 73-84.

24. Lescher F, Camblong H, Curea O, Briand R. LPV Control of wind turbines for fatigue loads reduction using intelligent microsensors. In Proceedings of the IEEE American Control Conference, Vol. 7, New York, U.S.A. 2007; 6061-6066.

25. de Corcuera AD, Pujana-Arrese A, Ezquerra JM, Milo A, Landaluze J. Linear Models-based LPV modelling and control for wind turbines. Wind Energy 2015; 18: 1151-1168.

26. van der Hooft EL, van Engelen TG, Pierik JTG, Schaak P, Real-time process simulator for evaluation of wind turbine systems: modeling and implementation, Technical Report (ECN-E-07-046), Energy Research Centre of the Netherlands (ECN), 2007.

27. van Engelen TG, van der Hooft EL. Dynamic inflow compensation for pitch controlled wind turbines. In Proceedings of the European Wind Energy Conference, London, U.K, 2004.

28. van Engelen TG. Design model and load reduction assessment for multi-rotational mode individual pitch Ccntrol (higher harmonics control). In Proceedings of the European Wind Energy Conference, Athens, Greece, 2006.

29. Ragan P, Manuel L. Comparing estimates of wind turbine fatigue loads using time-domain and spectral methods. Wind Engineering 2007; 31(2): 83-99. 
30. Duckwitz D, Shan M. A survey on control concepts for the reduction of tower loads (Conference Poster). In The Science of Making Torque from Wind (TORQUE 2012) Conference, 2012.

31. Duckwitz D, Shan M. Active tower damping and pitch balancing - design, simulation and fieldtest. In Proceedings of The Science of Making Torque from Wind (TORQUE 2012) Conference, Oldenburg, Germany, 2012.

32. Johnson SJ, van Dam CP, Berg DE, Active Load Control Techniques for Wind Turbines, Technical Report (No. SAND2008-4809), SANDIA National Laboratories, 2008.

33. Kanev S, Savenije F, Wouters D, Engels W, Control Design Tool Upgrade (CDTup) Project: Final Report, Technical Report (No. ECN-X-12-086), Energy Research Centre of the Netherlands (ECN), 2012.

34. Shan M, Jacobsen J, Adelt S. Field testing and practical aspects of load reducing pitch control systems for a 5 MW offshore wind turbine. In Proceedings of the 2013 European Wind Energy Association Event, Vienna, Austria, 2013; 632-641.

35. Anderson BDO, Moore JB. Optimal Control: Linear Quadratic Methods. Dover Publications: New York U.S.A, 2007.

36. Åstrom KJ, Wittenmark B. Computer-Controlled Systems: Theory and Design. Dover Publications: New York, U.S.A, 2011.

37. Kanev S, Schuurmans J, Rutterman R, Nguyen E. Towards new industrial software for advanced wind turbine control. In Proceedings of the European Wind Energy Conference, Brussels, Belgium, 2011.

38. Ben-Tal A, Nemirovski A. Lecture Notes on Modern Convex Optimization: Analysis, Algorithms and Engineering Applications. U.S.A: SIAM Philadelphia, 2001.

39. Boyd S, Vandenberghe L. Convex Optimization. Cambridge University Press: Cambridge, U.K, 2004.

40. Friedland B. Control System Design: An Introduction to State-Space Methods. Dover Publications: New York, U.S.A, 2005.

41. Tóth R, Lovera M, Heuberger PSC, Corno M, Van den Hof PMJ. On the discretization of linear fractional representations of LPV systems. IEEE Transactions on Control Systems Technology 2012; 20(6): 1473-1489.

42. Courties C, Bernussou J. LPV Control by dynamic output feedback. In Proceedings of the American Control Conference, 1999; 2267-2271.

43. de Souza CE, Trofino A. Gain-scheduled $\left(\mathcal{H}_{2}\right)$ controller synthesis for linear parameter-varying systems via parameter-dependent Lyapunov functions. International Journal of Robust and Nonlinear Control 2006; 16(1): 243-257.

44. de Souza CE, Trofino A, de Oliveira J. Parametric Lyapunov function approach to $\left(\mathcal{H}_{2}\right)$ analysis and control of linear parameter-dependent systems. In Proceedings of the IEEE Conference on Control Theory and Applications 2003: 501-508.

45. Scherer CW. Robust generalized $\left(\mathcal{H}_{2}\right)$ control for uncertain and LPV systems with general scalings. In Proceedings of the IEEE Conference on Decision and Control, Kobe, Japan, 1996; 3970-3975.

46. Feron E, Balakrishnan V, Boyd S, El Ghaoui L. Numerical methods for $\left(\mathcal{H}_{2}\right)$-related problems. Inproceedings of the American Control Conference, Vol. 4, Chicago, U.S.A. 1992; 2921-2922.

47. Boyd S, El Ghaoui L, Feron E, Balakrishnan V. Linear Matrix Inequalities in System and Control Theory: SIAM Philadelphia, U.S.A, 1994.

48. Wu F, Yang XH, Packard A, Becker G. Induced $\left(\mathcal{L}_{2}\right)$-norm control for LPV systems with bounded parameter variations rates. International Journal of Robust and Nonlinear Control 1996; 6: 983-998.

49. Gahinet P, Nemirovski A, Laub AJ, Chilali M. The LMI control toolbox. In Proceedings of the IEEE Conference on Decision and Control, Florida, U.S.A. 1994; 2038-2041.

50. Lindenburg C, Comparison of PHATAS versions and the wind turbine module, Technical Report (No. ECN-E-11-066), Energy Research Centre of the Netherlands (ECN), 2011.

51. Jonkman J, Butterfield S, Musial W, Scott G, Definition of a 5 MW Reference Wind Turbine for Offshore System Development, Technical Report (No. NREL/TP-500-38060), National Renewable Energies Laboratory (NREL), 2009. 